\title{
Diagnostic value of end tidal capnography in patients with hyperglycemia in the emergency department
}

\author{
Ralphe Bou Chebl ${ }^{1,2^{*}}$, Bryan Madden², Justin Belsky ${ }^{3}$, Elie Harmouche ${ }^{2}$ and Lenar Yessayan ${ }^{4,5}$
}

\begin{abstract}
Background: Diabetic Ketoacidosis (DKA) is a potentially life-threatening emergency that requires prompt diagnosis and treatment. In paediatric populations an end tidal capnography value greater than $36 \mathrm{mmHg}$ was found to be $100 \%$ sensitive in ruling out DKA.

Methods: A cross sectional observational study of adults $\geq 17$ years of age presenting to the emergency department between January 2014 and May 2014 with glucose > 550 mg/dL. In all patients, nasal capnography and venous blood gas analysis were performed prior to any insulin or intravenous fluid administration. The diagnosis of DKA was based on the presence of anion gap metabolic acidosis, hyperglycaemia and ketonemia. The overall diagnostic performance (area under the curve $[A \cup C]$ ), sensitivity, specificity and likelihood ratios at different end tidal $\mathrm{CO}_{2}\left(\mathrm{ETCO}_{2}\right)$ cut-offs were determined.

Results: 71 patients were enrolled in the study of which 21 (30\%) met the diagnosis of DKA. The area under the curve for $\mathrm{ETCO}_{2}$ was 0.95 with a $95 \% \mathrm{Cl}$ of 0.91 to 0.99 . Test sensitivity for DKA at $\mathrm{ETCO}_{2}$ level $\geq 35 \mathrm{mmHg}$ was $100 \%$ (95\% Cl, 83.9-100). An ETCO 2 level $\leq 21$ mmHg was $100 \%$ specific (95 \% Cl, 92.9-100.0) for DKA.

Conclusion: Nasal capnography exhibits favourable diagnostic performance in detecting patients with or without DKA among those who present to the emergency department with a glucometer reading $>550 \mathrm{mg} / \mathrm{dL}$.
\end{abstract}

Keywords: Capnography, Diabetic ketoacidosis, Emergency department

\section{Background}

Diabetes mellitus is the most common endocrine disease in the world. Acute complications of diabetes include severe hyperglycaemia, diabetic ketoacidosis (DKA) and hyperosmolar hyperglycaemic state (HHS). DKA is a potentially life-threatening emergency characterized by hyperglycaemia, metabolic acidosis and ketonemia along with severe electrolyte abnormalities [1]. HHS is defined as a state of severe hyperglycaemia without ketosis, with a glucose $>600 \mathrm{mg} / \mathrm{dL}$ but with levels frequently exceeding $1000 \mathrm{mg} / \mathrm{d}$. HHS is also characterized by neurological impairment, with the presentation ranging from altered mental status to stupor. All hyperglycaemic states require fluid resuscitation. The administration of

\footnotetext{
* Correspondence: rb94@aub.edu.lb

${ }^{1}$ Department of Emergency Medicine, American University of Beirut, Beirut, Lebanon

${ }^{2}$ Department of Emergency Medicine, Henry Ford Hospital, Detroit, MI, USA Full list of author information is available at the end of the article
}

regular insulin to DKA patients via continuous intravenous infusion or by frequent subcutaneous or intramuscular injections is the mainstay of treatment and is needed to stop the breakdown of fatty acids into ketones and to revert the acidotic state. While insulin is not harmful in severe hyperglycemia, patients may achieve euglycaemia with fluid resuscitation alone and an insulin drip is not needed [2]. Capnography provides an indirect means to detect metabolic acidosis. It monitors the partial pressure of carbon dioxide $\left(\mathrm{CO}_{2}\right)$ in respiratory gases. The partial pressure of $\mathrm{CO}_{2}$ at end expiration is termed end tidal $\mathrm{CO}_{2}\left(\mathrm{ETCO}_{2}\right)$ [3]. In response to the decreased serum $\mathrm{pH}$ that is associated with a decrease in serum bicarbonate, an increase would occur in alveolar minute ventilation with a corresponding decrease in arterial $\mathrm{CO}_{2}$ tension. Because $\mathrm{ETCO}_{2}$ closely approximates arterial $\mathrm{CO}_{2}$ tension, it can potentially be used to predict the presence or absence of DKA. Obtaining blood tests 
may be time-consuming. A rapid non-invasive sensitive screening tool that rules out DKA in patients with severe hyperglycaemia may avoid unnecessary administration of insulin infusion as part of initial therapy. Similarly, a non-invasive test with high specificity in identifying those with DKA; may expedite prompt diagnosis and treatment of DKA.

\section{Goals of this investigation}

In our emergency department, patients found to have blood glucose $>550 \mathrm{mg} / \mathrm{dL}$, regardless of clinical status, are taken to our high acuity area. This triage process often leads to overcrowding the critical area and overwhelming the medical team. For this subset of patients, we wished to investigate the diagnostic characteristics of $\mathrm{ETCO}_{2}$ to assess patients for DKA versus severe hyperglycaemia. We hypothesized that $\mathrm{ETCO}_{2}$ exhibited at least a "fair discriminatory power" (i.e., $0.7<\mathrm{AUC}<0.8$ ) [4] for DKA in patients with blood glucose $>550 \mathrm{mg} / \mathrm{dL}$. At $80 \%$ power, alpha $=0.05$, a ratio of sample sizes in negative to positive groups with DKA of 4 , and an area under the receiver operating curve (ROC) of 0.75 (fair discriminatory power) that is significant from the null hypothesis value of 0.5 (no discriminatory power), a minimum of 64 patients were required for the study. To include at least this number of patients, a 6-month inclusion period was considered. The objectives of this study were to characterize the overall diagnostic performance (area under the curve $[\mathrm{AUC}]$ ) of $\mathrm{ETCO}_{2}$ in patients with blood glucose $>550 \mathrm{mg} / \mathrm{dL}(30.6 \mathrm{mmol} / \mathrm{L})$; to determine the sensitivity, specificity, likelihood ratios at different $\mathrm{ETCO}_{2}$ cut-offs; and to establish the degree of correlation of both venous $\mathrm{pH}$ and serum bicarbonate with $\mathrm{ETCO}_{2}$, and between venous $\mathrm{pH}$ and serum bicarbonate.

\section{Methods}

\section{Study design and setting}

Cross sectional observational study was conducted at a single emergency department in an urban tertiary care centre in Detroit, Michigan, between January 2014 and May 2014. Given that there was no harm or injury in applying an end tidal monitor to patients' nasal cannula, a waiver of informed consent was granted. This study was approved by our hospital's institutional review board (IRB \#8560).

\section{Selection of participants}

As per our emergency department's protocol, patients with a known history of diabetes, or patients presenting with specific complaints (generalized weakness, vomiting, diaphoresis, altered mental status, polyuria) are screened at triage with a point of care glucose measurement. Inclusion criterion included a point of care glucometer (Accu-Chek Inform II, Roche, Basel, Switzerland) reading of "high" on presentation which corresponds to a glucose level $>550 \mathrm{mg} / \mathrm{dL}$. Exclusion criteria consisted of receipt of intravenous fluids or insulin prior to the application of nasal capnography.

\section{Methods and measurements}

Patients were approached and identified by the nursing staff in triage. Patient recruitment and enrolment occurred at all times throughout the duration of the study. All Eligible patients had nasal $\mathrm{CO}_{2}$ sampling cannula placed by trained medical personnel while they were being placed on the monitor and having their initial vital signs taken. The cannula was attached to a capnograph/ capnometer (Microstream $\mathrm{EtCO}_{2}$ consumable, Smart Capnoline, Philips Healthcare, Andover, MA). A single $\mathrm{ETCO}_{2}$ value was documented when a stable waveform was recorded on the monitor (Philips, intellivue MX700, Philips healthcare, Andover, MA). Venous blood was collected into collecting tubes for electrolytes, glucose, beta hydroxybutyrate $(\Omega \mathrm{HB})$, and complete blood count. No time delays between the blood draws and the application of the end tidal capnography. A venous blood gas (VBG) sample was then collected in an arterial blood collection syringe, sent to the laboratory over ice and analysed by blood gas analyser (ABL800 Flex, Radiometer America Inc., Westlake, $\mathrm{OH})$. Measurements of interest were $\mathrm{pH}$ and $\mathrm{CO}_{2}$ obtained from the VBG sample and measured serum bicarbonate ( $\mathrm{HCO} 3), ß \mathrm{HB}$, and blood glucose from routine analysis of venous blood. Capnography was compared to a reference standard for diagnosing DKA defined as a triad of hyperglycaemia $>250 \mathrm{mg} / \mathrm{dL}$, high anion gap metabolic acidosis and ketonemia [5]. Ketonemia was defined as $ß \mathrm{HB}$ level $>3 \mathrm{mmol} / \mathrm{l}$. The high anion gap acidosis was defined as having an anion gap $>12$ with either one of the following criteria; a serum bicarbonate $\leq 18 \mathrm{mmol} / \mathrm{L}$ or a blood $\mathrm{pH}<7.30$. Given that the inclusion criteria was a glucose $>550 \mathrm{mg} / \mathrm{dL}$, all patients met the hyperglycaemia criterion.

\section{Analysis}

Statistical analysis was performed using SAS software version 9.3 (SAS Institute, Inc., Cary, NC) and ROCs were performed using MedCalc Statistical Software version 13.2.2 (MedCalc Software bvba, Ostend, Belgium). Categorical variables were presented as frequencies and percentages. Continuous variables were intentionally presented as median, interquartile range (IQR), minimum and maximum to appreciate the spread of the data. Mann Whitney rank sum test was used for group comparisons for continuous data. Categorical data comparisons were performed using chi-square test. Pearson coefficients were used to evaluate the correlation 
Table 1 Patient characteristics

\begin{tabular}{llll}
\hline & DKA $(n=21)$ & Non-DKA $(n=50)$ & $P$ value \\
\hline Age (years) median (IQR) & $44(29-48)$ & $53(41-62)$ & 0.02 \\
Males $n(\%)$ & $12(57)$ & $27(54)$ & 0.03 \\
IDDM $n(\%)$ & $12(57)$ & $12(24)$ & 0.02 \\
CKD $n(\%)$ & $2(9)$ & $12(24)$ & 0.13 \\
\hline
\end{tabular}

DKA diabetic ketoacidosis, CKD chronic kidney disease (baseline glomerular filtration rate less than $60 \mathrm{ml} / \mathrm{min}$ ), $I Q R$ interquartile range

between $\mathrm{ETCO}_{2}$ and other arterial blood measurements and between venous $\mathrm{pH}$ and serum bicarbonate. Sensitivities and specificities were calculated at different levels of $\mathrm{ETCO}_{2}$. For all analyses, $P<.05$ was considered significant.

\section{Results}

\section{Characteristics of study patients}

Seventy-one consecutive patients were enrolled in the study. The median age was 48.0 years (IQR 39-58) and 39 (55 \%) were male. All patients had a known history of diabetes. Twenty-one patients had DKA (prevalence $30 \%)$. There was a significantly higher percentage of insulin dependent diabetic patients in the DKA group (57\% versus $24 \%$ ). Patient demographics, by presence or absence of DKA are presented in Table 1. Laboratory and diagnostic values are presented in Table 2. ETCO values ranged between 5 and $35 \mathrm{mmHg}$ in patients with DKA. Patients with DKA had higher median levels of glucose and $\mathrm{BHB}$ and lower partial pressure of $\mathrm{CO}_{2}$ $\left(\mathrm{pCO}_{2}\right), \mathrm{ETCO}_{2}$, serum bicarbonate, and $\mathrm{pH}$.

\section{Main results}

The diagnostic performance of $\mathrm{ETCO}_{2}$ in diagnosing DKA is represented in the ROC in Fig. 1. The area under the curve [AUC] was 0.95 (95\% CI, 0.91 to 0.99). Test sensitivity at $\mathrm{ETCO}_{2}$ level $\geq 35 \mathrm{mmHg}$ was $100 \%$ (95\% CI, 83.9-100) and specificity was 68 (95\% CI, 5380). At an $\mathrm{ETCO}_{2}$ level less than or equal to $21 \mathrm{mmHg}$ specificity was $100 \%$ (95\% CI, 92.9-100.0) and sensitivity was $57 \%$ (95\% CI, 34-78). Table 3 provides the sensitivities and specificities for DKA at different $\mathrm{ETCO}_{2}$ cut-offs.

Finally, there was a strong correlation between $\mathrm{ETCO}_{2}$ and serum bicarbonate levels $(r=0.88, P<.0001)$, coefficient of determination $r^{2}=0.76$ (Fig. 2); and between $\mathrm{ETCO}_{2}$ and VBG pH $(r=0.75, P<.0001)$, coefficient of determination $\mathrm{r}^{2}=0.56$ (Fig. 3). There was also a strong correlation between the VBG $\mathrm{pH}$ and measured serum bicarbonate $(r=0.84, P<0.0001)$, coefficient of determination $r^{2}=0.70$.

\section{Discussion}

DKA is one of the few endocrine emergencies. It is characterized by hyperglycaemia, ketonemia and metabolic acidosis along with electrolyte abnormalities. It is a condition that should be readily recognized and promptly treated. The mainstay of medical treatment is intravenous fluid rehydration, insulin replenishment through an intravenous drip, electrolyte correction and identification of the cause of the ketotic state [1].

DKA and severe hyperglycaemia are distinct clinical entities with different treatments. Although the mainstay treatment is fluid resuscitation, patients with DKA require insulin to reverse their ketotic state. Patients with severe hyperglycaemia without ketosis require fluid resuscitation first and foremost; they may or may not need insulin, and those who do infrequently require continuous insulin infusion [6]. We sought to study the test characteristics of $\mathrm{ETCO}_{2}$ in those presenting with blood sugar greater than $550 \mathrm{mg} / \mathrm{dL}$ in an attempt to identify thresholds which may help identify those with or without concomitant DKA.

In this cohort of adult patients presenting with high glucose, $\mathrm{ETCO}_{2}$ exhibited a good diagnostic performance (AUC 0.95), $95 \%$ CI, 0.91 to 0.99. DKA was highly prevalent in patients with glucose $>550 \mathrm{mg} / \mathrm{dL}$ (prevalence $30 \%$ ). At $\mathrm{ETCO}_{2} \leq 21 \mathrm{mmHg}$, DKA was ruled in (specificity $100 \%$ ) At $\mathrm{ETCO}_{2} \geq 35 \mathrm{mmhg}$, DKA was ruled out (sensitivity of $100 \%$ ). Furthermore, there was a strong correlation between $\mathrm{ETCO}_{2}$ and $\mathrm{PH}$ as well as bicarbonate, which would suggest that $\mathrm{ETCO}_{2}$ could be useful in identifying DKA patients presenting with

Table 2 Outcome variables

\begin{tabular}{llll}
\hline & DKA $(n=21)$ & Non-DKA $(n=50)$ & $P$ value \\
\hline Glucose $(\mathrm{mg} / \mathrm{dL})$ & $804(650-897)$ & $592(564-675)$ & 0.003 \\
ETCO2 $(\mathrm{mm} \mathrm{Hg})$ & $17(9-27)$ & $37(34-41)$ & $<0.001$ \\
pH & $7.16(7.01-7.27)$ & $7.38(7.33-7.43)$ & $<0.001$ \\
pCO2 $(\mathrm{mm} \mathrm{Hg})$ & $25(15.0-29.7)$ & $41.75(37.0-47.0)$ & $<0.001$ \\
Bicarbonate $(\mathrm{mmol} / \mathrm{L})$ & $9(5-15)$ & $24(22-25)$ & $<0.001$ \\
Beta-Hydroxybutyrate $(\mathrm{mmol} / \mathrm{L})$ & $11.35(7.9-14.0)$ & $0.37(0.17-1.21)$ & $<0.001$ \\
\hline
\end{tabular}

Data presented as median (IQR). ETCO2 end tidal carbon dioxide, $p C O 2$ partial pressure of carbon dioxide, CKD chronic kidney disease with baseline glomerular filtration rate less than $60 \mathrm{ml} / \mathrm{min}, I D D M$ insulin dependent diabetes mellitus 


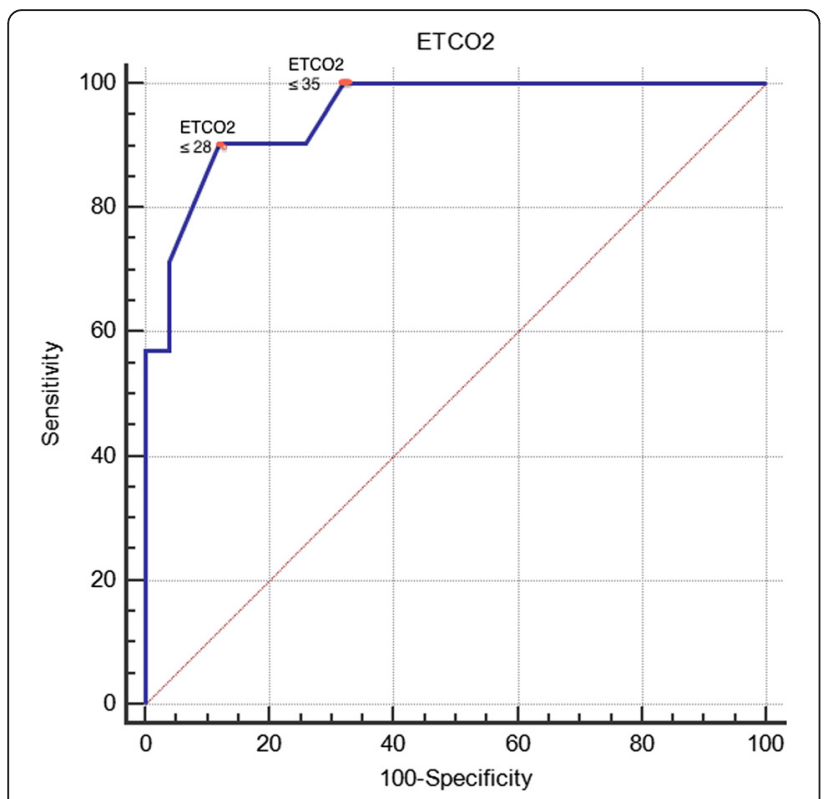

Fig. 1 ROC curve showing overall diagnostic accuracy of ETCO2

remarkably low $\mathrm{PH}$ levels and would allow faster and more aggressive care.

Our study differs from other studies in several ways. First, our cohort consisted of patients with blood glucose $>550 \mathrm{mg} / \mathrm{dl}$ measured by glucometer. Second, we used VBG pH to diagnose acidemia. Several studies have shown that venous $\mathrm{pH}$ and $\mathrm{pCO}_{2}$ correlated highly with arterial $\mathrm{pH}$ and $\mathrm{pCO}_{2}$ [7-9]. In patients with DKA, Ma et al. demonstrated that venous and arterial $\mathrm{pH}$ have also high agreement (mean bias $-0.015 \pm 0.006 \mathrm{pH}$ units) [10]. In this study, we also found a strong correlation between the VBG $\mathrm{pH}$ and measured serum bicarbonate $(R$ $=0.8395, P<0.0001)$. Therefore, it is reasonable to replace the arterial blood gas with venous blood gas in the evaluation of a patient with DKA. Third, we used $3 \mathrm{HB}$ level $>3 \mathrm{mmol} / \mathrm{L}$ to diagnose ketonemia. Although the
American Diabetes Association (ADA) [11] states that urinary ketones are not reliable and that measurement of $ß \mathrm{HB}$ is preferred, no specific diagnostic cut-offs are mentioned. Studies that examined the correlation between serum bicarbonate and $\mathrm{BHB}$ in DKA patients determined that a serum $B \mathrm{HB}$ level $<3 \mathrm{mmol} / \mathrm{L}$ does not correlate with an acidotic state [12, 13]. Fourth, our study was conducted in adult patients. Most of the studies that have evaluated the relationship between $\mathrm{ETCO}_{2}$ and acidosis were in pediatric patients with type I diabetes [14-16]. These studies have shown a very high diagnostic accuracy in pediatric patients. Both Fearon et al. [14] and Gilhotra et al. [16] showed $\mathrm{ETCO}_{2}$ values less than $29 \mathrm{mmHg}$ could identify DKA with a specificity of 100 and $91 \%$, respectively, whereas no patient with $\mathrm{ETCO}_{2}$ values $>35 \mathrm{mmHg}$ had DKA (sensitivity $100 \%$ ). In contradistinction, in this study, the corresponding specificity at $\mathrm{ETCO}_{2}$ values $<29 \mathrm{mmHg}$ was only $88 \%$ (95\% CI, 75-95), however, the sensitivity at $\mathrm{ETCO}_{2}$ values $>35 \mathrm{mmHg}$ was also $100 \%$ (95\% CI, 84-100). The different diagnostic accuracies at the same $\mathrm{ETCO}_{2}$ cut-offs between our study and the paediatric studies may be secondary to different spectrum and distribution of acute or chronic acid base disorders in adults compared to the paediatric population or secondary to statistical variation.

To our knowledge, only one study by Soleimanpour et al. has evaluated the utility of $\mathrm{ETCO}_{2}$ in adults as a screening tool for DKA with blood glucose $>250 \mathrm{mg} / \mathrm{dL}$ and clinical symptoms of DKA [17]. The study was conducted in an emergency department in Iran. The mean glucose levels were $458 \mathrm{mg} / \mathrm{dL}$ in DKA patients and $362 \mathrm{mg} / \mathrm{dL}$ in non-DKA patients. The Soleimanpour study showed that $\mathrm{ETCO}_{2}$ had a uniquely high diagnostic accuracy (AUC 0.963 ) overall, and at $\mathrm{ETCO}_{2}$ cut-off of $24.5 \mathrm{mmHg}$; it was highly sensitive (90\%) and specific (90\%). The diagnostic performance of our test as measured by AUC was very high (AUC 0.95). Specificity at

Table 3 Sensitivities and specificities of end tidal capnography

\begin{tabular}{lcccccccc}
\hline Criterion & Sensitivity & $95 \% \mathrm{Cl}$ & Specificity & $95 \% \mathrm{Cl}$ & \multicolumn{1}{c}{$+\mathrm{LR}$} & $95 \% \mathrm{Cl}$ & $-\mathrm{LR}$ & $95 \% \mathrm{Cl}$ \\
\hline$\leq 21$ & 57.14 & $34-78.2$ & 100.00 & $92.9-100.0$ & 0.00 & & 0.43 & $0.3-0.7$ \\
$\leq 23$ & 57.14 & $34-78.2$ & 98.00 & $89.4-99.9$ & 28.57 & $4.0-206.0$ & 0.44 & $0.3-0.7$ \\
$\leq 25$ & 57.14 & $34-78.2$ & 96.00 & $86.3-99.5$ & 14.29 & $3.5-58.4$ & 0.45 & $0.3-0.7$ \\
$\leq 26$ & 71.43 & $47.8-88.7$ & 96.00 & $86.3-99.5$ & 17.86 & $4.5-71.3$ & 0.30 & $0.2-0.8$ \\
$\leq 27$ & 85.71 & $63.7-97.0$ & 90.00 & $78.2-96.7$ & 8.57 & $3.7-20.0$ & 0.16 & $0.06-0.5$ \\
$\leq 28$ & 90.48 & $69.9-98.8$ & 88.00 & $75.7-95.5$ & 7.54 & $3.5-16.2$ & 0.11 & $0.03-0.4$ \\
$\leq 31$ & 90.48 & $69.9-98.8$ & 86.00 & $73.3-94.2$ & 6.46 & $3.2-13.0$ & 0.11 & $0.03-0.4$ \\
$\leq 32$ & 90.48 & $69.9-98.8$ & 84.00 & $70.9-92.8$ & 5.65 & $3.0-10.8$ & 0.11 & $0.03-0.4$ \\
$\leq 33$ & 90.48 & $69.9-98.8$ & 80.00 & $66.3-90.0$ & 4.52 & $2.6-8.00$ & 0.12 & $0.03-0.4$ \\
$\leq 34$ & 90.48 & $69.9-98.8$ & 74.00 & $59.7-85.4$ & 3.48 & $2.1-5.7$ & 0.13 & $0.03-0.5$ \\
$\leq 35$ & 100.00 & $83.9-100$ & 68.00 & $53.3-80.5$ & 56.8 & $2.1-4.7$ & 0.00 & \\
\hline
\end{tabular}




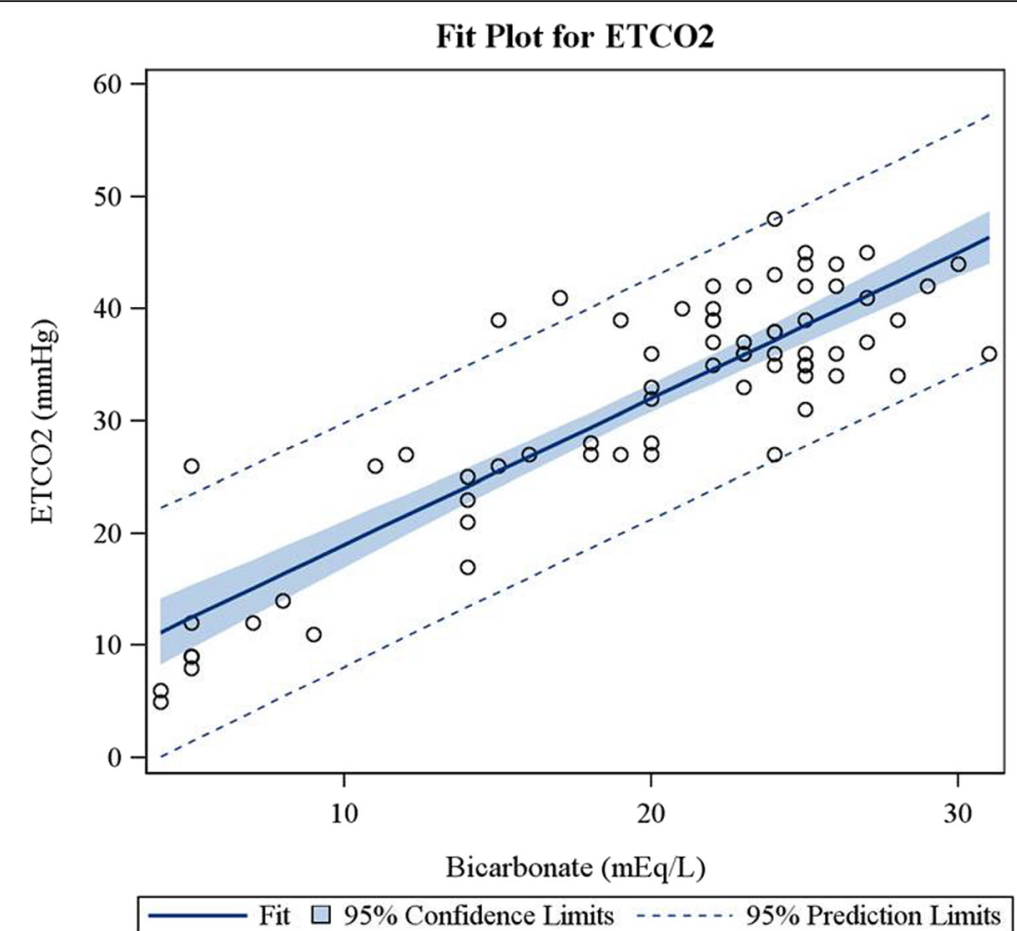

Fig. 2 ETCO2 and bicarbonate correlation fit plot

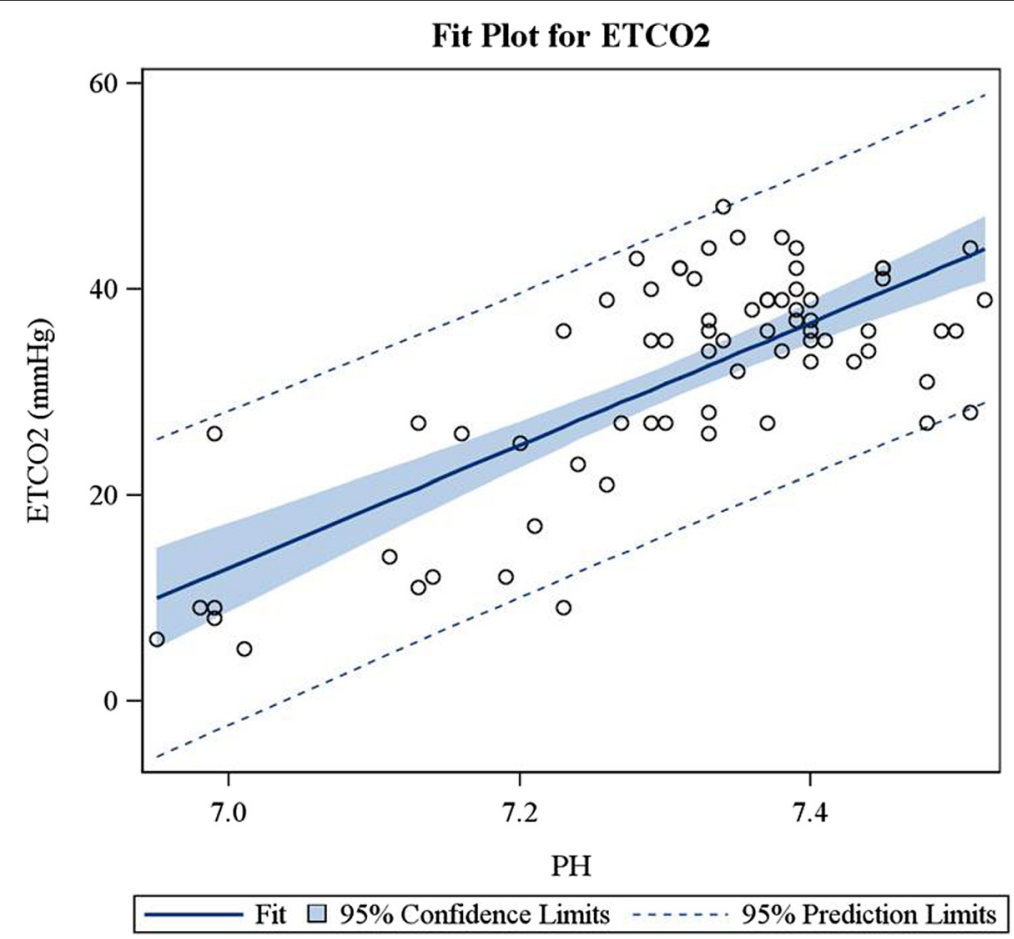

Fig. 3 ETCO2 and venous blood gas PH correlation fit plot 
$\mathrm{ETCO}_{2}$ cut-off of 24.5 in our study was slightly higher (96\% vs. $90 \%$ ); however our study did not demonstrate a high sensitivity at this $\mathrm{ETCO}_{2}$ cut-off (sensitivity $57 \%$ vs. $90 \%)$. Several patients in our cohort had DKA at higher $\mathrm{ETCO}_{2}$ levels than 24.5 either because of chronic respiratory acidosis disorders or because of coexisting metabolic alkalosis.

\section{Limitations}

Our study has some limitations. First, the results can only be applied to patients with blood glucose $>550 \mathrm{mg} /$ $\mathrm{dL}(30.6 \mathrm{mmol} / \mathrm{L})$. It is important to note that patients with blood glucose level $<550 \mathrm{mg} / \mathrm{dL}$ can be in DKA, however we chose not to look at this subset of population since it was studied earlier in the literature and because our main objective was to check the test characteristics of the test among patients with severe hyperglycemia [17]. A non-invasive test with a strong positive predictive value for DKA would also be useful to identify and urgently treat those with glucose levels that might not otherwise be concerning. Second, although the study was adequately powered to show that the AUC was $>0.50$, it remains a relatively small study with wide CIs around the sensitivity and specificity of any of the cut-off points. A more precise estimate of these cut-off points can be obtained by a larger sample size. Third, it would have been helpful to see the AUC for respiratory rate and compare it to the AUC for $\mathrm{ETCO}_{2}$ and perform a cost analysis for the use of $\mathrm{ETCO}_{2}$, however, this was not the aim of this study and it is our hope that this study can stimulate future research studies. Finally, It would also have been important to provide the data on other potential confounders of $\mathrm{ETCO}_{2}$ within our patient population, however a significant amount of our patients did not have any formal diagnosis of Chronic Obstructive Pulmonary Disease (COPD) or any Pulmonary Function Tests (PFTs) in our records.

\section{Conclusion}

This single centre study suggests that end tidal capnography in adults presenting to the emergency department with glucose levels $>550 \mathrm{mg} / \mathrm{dL}$ may useful in identifying and ruling out DKA. The external validity of the results should be confirmed by further prospective studies.

\section{Ethics}

This study was approved by Henry Ford Hospital's institutional review board (IRB \#8560). All procedures performed in studies involving human participants were in accordance with the ethical standards of the institutional review board at our institution and with the 1964
Helsinki declaration and its later amendments or comparable ethical standards.

\section{Consent}

A waiver of informed consent was granted by our IRB. The authors have obtained consent to publish data from participants.

\section{Competing interests}

The authors declare that they have no competing interests.

\section{Authors' contributions}

$R B$ and $L Y$ designed the study. RB, BM, JB and $E H$ acquired the data. $L Y$ and ZA analysed the data, RB, LY, GA and BJ wrote the manuscript and revised it. All authors read and approved the final manuscript.

\section{Author details}

${ }^{1}$ Department of Emergency Medicine, American University of Beirut, Beirut, Lebanon. ${ }^{2}$ Department of Emergency Medicine, Henry Ford Hospital, Detroit, MI, USA. ${ }^{3}$ Department of Emergency Medicine, Massachusetts General Hospital, Boston, MA, USA. ${ }^{4}$ Division of Nephrology and Hypertension, Henry Ford Hospital, Detroit, MI, USA. ${ }^{5}$ Division of Pulmonary and Critical Care Medicine, Henry Ford Hospital, Detroit, MI, USA.

Received: 18 October 2015 Accepted: 22 January 2016

Published online: 29 January 2016

\section{References}

1. Kitabchi AE, Nyenwe EA. Hyperglycemic crises in diabetes mellitus: diabetic ketoacidosis and hyperglycemic hyperosmolar state. Endocrinol Metab Clin North Am. 2006;35(4):725-51. doi:10.1016/j.ecl.2006.09.006.

2. McNaughton CD, Self WH, Slovis C. Diabetes in the emergency department: acute care of diabetes patients. Clin Diab. 2011;29(2):51-9. doi:10.2337/ diaclin.29.2.51

3. Ward KR, Yealy DM. End-tidal carbon dioxide monitoring in emergency medicine, part 1: basic principles. Acad Emerg Med. 1998;5(6):628-36.

4. Hanley JA, McNeil BJ. The meaning and use of the area under a receiver operating characteristic (ROC) curve. Radiology. 1982;143(1):29-36. doi:10. 1148/radiology.143.1.7063747.

5. Kitabchi AE, Wall BM. Diabetic ketoacidosis. Med Clin North Am. 1995;79(1): 9-37.

6. Ford W, Self WH, Slovis C, McNaughton CD. Diabetes in the emergency department and hospital: acute care of diabetes patients. Curr Emerg Hosp Med Rep. 2013;1(1):1-9. doi:10.1007/s40138-012-0007-x.

7. Byrne AL, Bennett M, Chatterji R, Symons R, Pace NL, Thomas PS. Peripheral venous and arterial blood gas analysis in adults: are they comparable? A systematic review and meta-analysis. Respirology. 2014. doi:10.1111/resp. 12225

8. Middleton P, Kelly AM, Brown J, Robertson M. Agreement between arterial and central venous values for $\mathrm{pH}$, bicarbonate, base excess, and lactate. Emerg Med J. 2006;23(8):622-4. doi:10.1136/emj.2006.035915.

9. Kelly AM, McAlpine R, Kyle E. Venous pH can safely replace arterial pH in the initial evaluation of patients in the emergency department. Emerg Med J. 2001;18(5):340-2.

10. Ma OJ, Rush MD, Godfrey MM, Gaddis G. Arterial blood gas results rarely influence emergency physician management of patients with suspected diabetic ketoacidosis. Acad Emerg Med. 2003;10(8):836-41.

11. Goldstein DE, Little RR, Lorenz RA, Malone Jl, Nathan DM, Peterson CM. American Diabetes Association: tests of glycemia in diabetes. Diabetes Care. 2004;27 Suppl 1:S91-3.

12. Sheikh-Ali M, Karon BS, Basu A, Kudva YC, Muller LA, Xu J, et al. Can serum beta-hydroxybutyrate be used to diagnose diabetic ketoacidosis? Diabetes Care. 2008;31(4):643-7. doi:10.2337/dc07-1683.

13. Taboulet $P$, Haas $L$, Porcher R, Manamani J, Fontaine JP, Feugeas JP, et al. Urinary acetoacetate or capillary beta-hydroxybutyrate for the diagnosis of ketoacidosis in the Emergency Department setting. Eur J Emerg Med. 2004; 11(5):251-8. 
14. Fearon DM, Steele DW. End-tidal carbon dioxide predicts the presence and severity of acidosis in children with diabetes. Acad Emerg Med. 2002;9(12): 1373-8.

15. Agus MS, Alexander JL, Mantell PA. Continuous non-invasive end-tidal CO2 monitoring in pediatric inpatients with diabetic ketoacidosis. Pediatr Diabetes. 2006;7(4):196-200. doi:10.1111/j.1399-5448.2006.00186.x.

16. Gilhotra Y, Porter P. Predicting diabetic ketoacidosis in children by measuring end-tidal $\mathrm{CO} 2$ via non-invasive nasal capnography. J Paediatr Child Health. 2007;43(10):677-80. do::10.1111/j.1440-1754.2007.01 186.x.

17. Soleimanpour H, Taghizadieh A, Niafar M, Rahmani F, Golzari SE, Esfanjani RM. Predictive value of capnography for suspected diabetic ketoacidosis in the emergency department. West J Emerg Med. 2013;14(6):590-4. doi:10.5811/westjem.2013.4.14296

Submit your next manuscript to BioMed Central and we will help you at every step:

- We accept pre-submission inquiries

- Our selector tool helps you to find the most relevant journal

- We provide round the clock customer support

- Convenient online submission

- Thorough peer review

- Inclusion in PubMed and all major indexing services

- Maximum visibility for your research

Submit your manuscript at www.biomedcentral.com/submit
Biomed Central 